\title{
Monitoramento e modelagem hidrológica de plano de infiltração construído em escala real
}

\section{Monitoring and modelling hydrologic of plan infiltration built in real scale}

\author{
Natalia Tecedor ; Ademir Paceli Barbassa ${ }^{2}$; Rodrigo Moruzzi ${ }^{3}$ e Luciana Márcia Gonçalves ${ }^{4}$ \\ ${ }^{1}$ Universidade Federal de São Carlos, Programa de Pós-Graduação em Engenharia Urbana, São Carlos - SP \\ natytecedor@hotmail.com \\ ${ }^{2,4}$ Universidade Federal de São Carlos, Departamento de Engenharia Civil, São Carlos - SP \\ barbassa@ufscar.br; lucianamg@ufscar.br
}

${ }^{3}$ Eng $^{\circ}$ Civil, Mestre e Doutor em Engenharia Civil, Professor do Curso de Engenharia Ambiental

DEPLAN/ICGE/UNESP, Rio Claro - SP

rmoruzzi@r.unesp.br

Recebido: 21/09/14 - Revisado: 13/11/14 - Aceito: 27/04/15

\begin{abstract}
RESUMO
Neste trabalho objetivou-se monitorar e modelar um plano de infiltração, construído em escala real, para manejo de águas pluviais com área de $3.001 \mathrm{~m}^{2}$. Foram monitorados a precipitação, a vazão de entrada e o nivel de água no interior do plano. Os equipamentos foram calibrados por meio de ensaios de campo com o esvaziamento controlado, por meio do descarregamento de 9,3 $\mathrm{m}^{3}$ de água em ensaios em triplicada. Foram monitorados 32 eventos de chuvas, sendo que destes 7 apresentaram registros de lâmina. O método de PULS foi empregado para modelagem. O modelo foi calibrado empregando condutividade hidráulica variável e constante. Na etapa de calibração os valores de $R^{2}$ médios foram de 0,75 e 0,72 respectivamente. Na validação obtiveram-se valores de $\mathrm{R}^{2}$ de 0,71 para duas condutividades. Os tempos de esvaziamento, em torno de 25 min, foram explicados pelas altas taxas e áreas de infiltração. Os valores médios observados de tempo de retardo variaram em média 5,9 min; de tempo de pico em média 28,3 min e do tempo de escoamento na superfície do plano em média 12,2 min. Os resultados indicaram que o método de PULS pode ser aplicado com restrições, tendo em vista as vazões iniciais calculadas pelo PULS foram maiores que as observadas. Tal fato foi atribuido aos baixos valores de lâminas de água registrados e as irregularidades topográficas no plano gramado, os quais incorreram em imprecisão na relação nivel de água e volume inicial.
\end{abstract}

Palavras Chave: Técnicas compensatórias. Plano de infiltração. Método de PULS

\begin{abstract}
The purpose of this study was to monitor and later model the flow along an infiltration plane, built in real scale, for rain water management area of $3001 \mathrm{~m}^{2}$. Rainfall, inflow and water depth of flow over the inclined plane were monitored. The equipment employed for measurement was calibrated in triplicate through field measurements under conditions of controlled drainage of $9.3 \mathrm{~m}^{3}$ volume of water poured over the plane. 32 events were monitored of which only seven produced measurable water depth. The inclined plane was calibrated by PULS' method considering constant and variable bydraulic conductivity obtaining mean $\mathrm{R}^{2}$ of 0.75 and 0.72 . In the validation process, $R^{2}$ of 0.71 was obtained for both cases. The drainage times of around 25 minutes were explained by the high rates of infiltration and the area of the plane. Observed average lag times was 5,9 min; times to peak. 28,3min and flow time over the plane from 12,2 min. It was observed that the initial calculated water levels were superior to those observed, because there were flooded area, but the depth-area-volume ratio calculated by PULS did not adhere with observed data.
\end{abstract}

Keywords: Compensatory techniques. Infiltration plan. Method PULS. 


\section{INTRODUÇÃO}

O processo de urbanização acelerado e desordenado implicou em grandes alterações no meio ambiente natural e nos processos hidrológicos, tais como a redução da interceptação, do armazenamento superficial e da infiltração e aumento do escoamento superficial, tornando insustentáveis os sistemas de micro e macro drenagem (BAPTISTA; NASCIMENTO; BARRAUD, 2011).

Como alternativa à abordagem higienista, na qual predominam os processos de afastamento e aceleração do escoamento gerado em superfícies impermeáveis, surgiu na Europa e América do Norte em meados de 1970 uma nova abordagem para lidar com essas alterações ambientais e hidrológicas, as chamadas tecnologias alternativas ou compensatórias. Tais técnicas buscam atenuar os efeitos da urbanização sobre os processos hidrológicos por meio da retenção e detenção dos volumes excedentes, minimizando a rápida transferência dessas águas para jusante (BAPTISTA; NASCIMENTO; BARRAUD, 2011).

Dentre as técnicas compensatórias existentes estão as estruturas de infiltração, definidas por Urbonas (1993) como locais permeáveis destinados à infiltração da água no solo, por meio de valas, faixas de proteção gramadas, pavimento poroso, trincheiras de percolação e bacias de infiltração.

Schueler (1987) ressalta que o bom desempenho dessas técnicas depende do tipo de solo, vegetação e da declividade da superfície que influenciarão na velocidade do escoamento. Martin, Ruperd e Legret (2006) destacaram a prevenção das inundações como principal vantagem do emprego dessas técnicas, o que supera em muito os incentivos econômicos necessários a sua implantação.

No Brasil, há estudos sobre essas técnicas, tais como apresentado por Lucas, Barbassa e Moruzzi (2013) e Souza (2002) com trincheiras de infiltração, Barbassa, Angelini Sobrinha e Moruzzi (2014) e Reis, Oliveira e Sales (2008) com poços de infiltração, dentre outros. Já no exterior, há várias pesquisas sobre o tema, com destaque às pesquisas realizadas na França: Bourgogne (2010), Dechesne, Barraud e Bardin (2005) e Fuamba et. al. (2010) e também Chahar, Graillot e Gaur (2012) no Canadá.

Especificamente, o plano de infiltração é uma técnica compensatória que pode ser definida como áreas permeáveis rebaixadas, com superfície coberta com gramados que recebem água pluvial advindas de áreas impermeáveis. Em relação à geometria, difere-se das demais por suas dimensões longitudinais não muito maiores que as transversais e por sua profundidade reduzida, conforme Baptista, Nascimento e Barraud (2011) e Moura (2005).

Embora existam estudos recentes sobre o funcionamento e modelagem de diversas técnicas compensatórias, existe grande carência de dados experimentais sobre planos de infiltração, tanto em relação ao seu funcionamento quanto à avaliação do comportamento hidrológico desses sistemas.

Assim, esse trabalho buscou um maior entendimento acerca do funcionamento e do comportamento hidrológico de um plano de infiltração, construído em escala real.

\section{OBJETIVO}

Monitorar e modelar hidrologicamente um plano de infiltração em escala real para manejo de água pluvial.

\section{METODOLOGIA}

\section{Local para implantação}

O plano de infiltração foi construído em bacia experimental localizada no Campus da UFSCar em São Carlos - SP, mais especificamente na zona norte da universidade, para manejo de água pluvial do prédio do Departamento de Fisioterapia, como ilustra a figura 1.

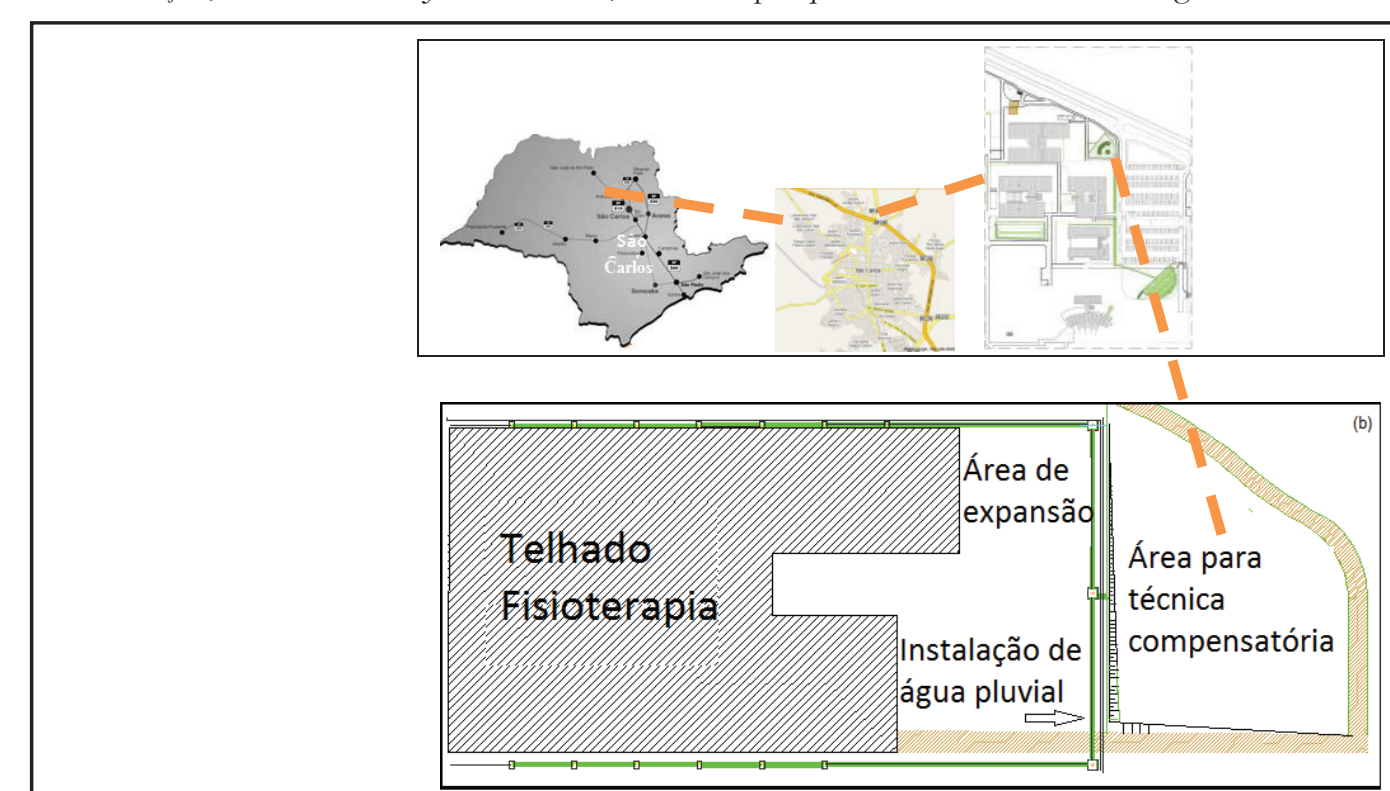

Figura 1 - (a) Localização da microbacia experimental e (b) edifício da Fisioterapia cujo escoamento pluvial será manejado. Fonte: Grupo G-Hidro 


\section{Caracterização do plano de infiltração}

Os ensaios de granulometria do solo local foram realizados conforme NBR 7181 (ABNT, 1984) e NBR 6502 (ABNT, 1995). Os ensaios de compactação do solo foram realizados em superfície, a 0,90 m do nível do terreno, segundo NBR 6457 (ABNT, 1986a) e a NBR 7182 (ABNT, 1986b). Os índices físicos foram determinados conforme recomendações constantes nas NBR 6457 (ABNT, 1986a) e a NBR 7182 (ABNT, 1986b). Devido ao alto grau de compactação superficial do solo, verificou-se a inviabilidade da infiltração. Ensaios de duplo anel confirmaram taxas de infiltração praticamente nulas antes da construção. Assim, previu-se a recuperação da capacidade de infiltração por meio do rebaixamento da superfície do terreno em 0,30 m para armazenamento de água e descompactação do solo com escarificação de $0,5 \mathrm{~m}$.

O dimensionamento foi feito com a taxa de $\mathrm{K}$ saturado constante de $125 \mathrm{~mm} / \mathrm{h}$ de local a $100 \mathrm{~m}$. Após procedimento de recuperação, feito durante a construção, as taxas de infiltração foram comparadas por meio dos resultados dos ensaios de duplo anel em triplicada. Assim, a taxa final foi determinada e denominada de condutividade hidráulica saturada (Ksat).

\section{Dimensionamento do plano de infiltração}

O plano de infiltração foi dimensionado para receber as águas do telhado, constituído de chapa galvanizada com área de $1.747 \mathrm{~m}^{2}$ somada à área de expansão entre o prédio e o dispositivo, de $1.254 \mathrm{~m}^{2}$, totalizando $3.001 \mathrm{~m}^{2}$. O volume armazenado (Smax) obtido por meio do método das chuvas foi determinado com emprego das Equações 1 e 2, conforme proposto por Baptista, Nascimento e Barraud (2011).

$$
\text { Smáx.=DP máx. }(q s, T) \text { x } A a
$$

Sendo, DPmáx. (qs,T) variável que corresponde à diferença máxima entre altura precipitada e altura infiltrada, e $A a$ corresponde à área de telhado efetiva. Adotou-se para o telhado C=0,95 (ASCE, 1969 apud TUCCI; PORTO; BARROS, 1995), período de retorno (Tr) de 10 anos e a equação de chuva da cidade de São Carlos-SP de Barbassa (1991), exposta a seguir:

$$
I=\frac{28,03 \cdot \operatorname{Tr}^{0,199}}{(D+16)^{0,936}}
$$

Sendo: $I$ a intensidade da precipitação $(\mathrm{mm} / \mathrm{min}) ; \mathrm{Tr}$ período de retorno (anos) e ta duração da precipitação (minutos).

A chuva de projeto possui intensidade de $41,1 \mathrm{~mm} / \mathrm{h}$, duração de 70 minutos e altura de água de $48,0 \mathrm{~mm}$. O volume de armazenamento calculado foi de $106,5 \mathrm{~m}^{3}$ e o volume disponível de $111,34 \mathrm{~m}^{3}$. Mostra-se na figura 2 parte (a) a planta do plano de infiltração na forma de arco de elipse, sendo a dimensão do semieixo maior de 25,85 metros e do semieixo menor de 19,90 metros. Tem-se na Figura 2 parte (b) o corte AA a partir da vista em planta do plano de infiltração, sendo a lâmina máxima de água de $0,59 \mathrm{~m}$.

\section{Funcionamento do Plano de Infiltração}

O funcionamento do Plano de Infiltração ocorre seguindo as seguintes etapas, em correspondência com a numeração indicada na figura $2-a$ : $1^{\text {a }}$ etapa - a água precipitada no telhado é dirigida pela instalação predial pluvial até uma canaleta; $2^{a}$ etapa - conexão da instalação pluvial com o plano e medição da vazão de entrada; $3^{a}$ etapa - a água então é direcionada para uma canaleta, preenchida com brita, com a função de distribuí-la e evitar caminho preferencial no plano; $4^{\mathrm{a}}$ etapa - após passar pela canaleta de brita $(0,4 \mathrm{~m}$ por $0,3 \mathrm{~m})$, a água é distribuída ao plano onde ficará armazenada temporariamente até sua infiltração no solo; $5^{\text {a }}$ etapa - caso necessário, o volume excedente da capacidade do plano de infiltração é esgotado pela crista do barramento.

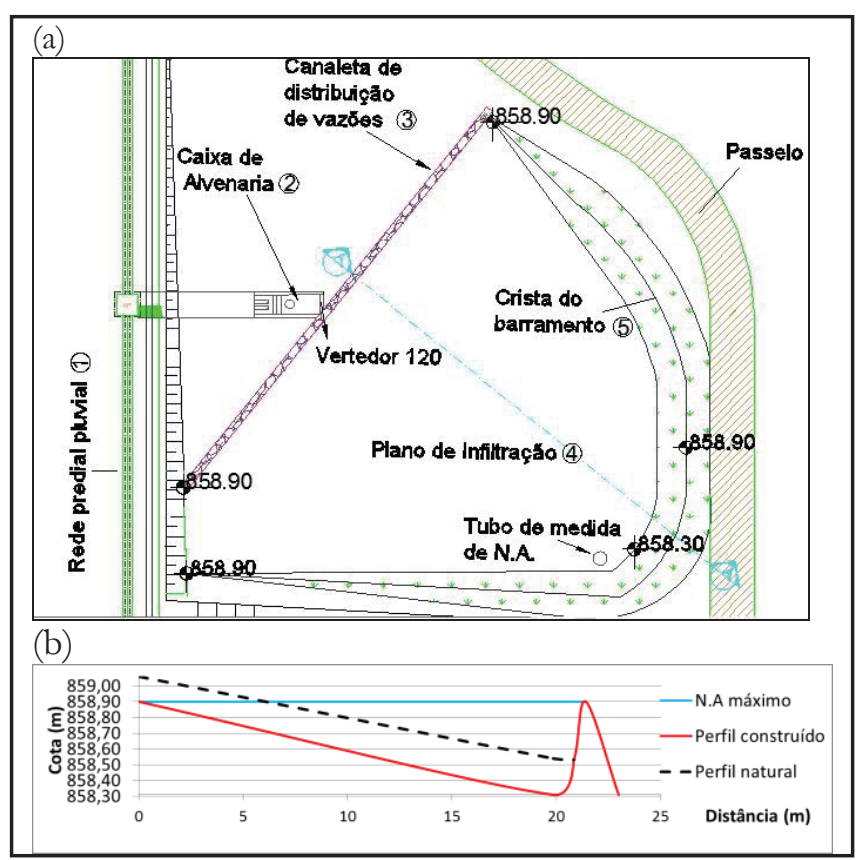

Figura 2 - (a) Plano de Infiltração gramado em planta e (b) no corte AA

\section{Monitoramento do Plano de Infiltração}

A precipitação foi monitorada com um pluviógrafo digital Campbell, instalado a $500 \mathrm{~m}$ do plano, configurado para registrar a intervalos de 1 minuto, com aferição apresentada em Lucas (2011).

Mediram-se as vazões de entrada no plano com vertedor triangular de parede fina de $120^{\circ}$ (Figura 4 parte b), conforme Equação 3 (LENCASTRE, 1983).

$$
Q_{e}=1,32 \cdot \tan (a / 2) \cdot h^{2,47}
$$

Sendo $Q_{\rho}$ a vazão $\left(\mathrm{m}^{3} / \mathrm{s}\right) ; \alpha$ o ângulo de abertura do vertedor em graus igual a $120^{\circ}$; $h$ a altura ou lâmina de água acima da crista do vertedor $(\mathrm{m})$.

$\mathrm{O}$ vertedor foi instalado em uma caixa de alvenaria de $2,8 \mathrm{~m}$ por 2,3 m, com tranquilizador, conforme figura 4 . 
As lâminas acima da crista do vertedor e no plano de infiltração foram medidas por dois sensores de nível OTT', modelo Orpheus Mini. Ambos os sensores foram instalados dentro de um tubo de PVC perfurado, sendo um deles instalado a 1,5 m, conforme Porto (2005), a montante do vertedor de $120^{\circ}$ (Figura 3), e o outro no ponto mais baixo do plano. Os sensores foram calibrados em laboratório com lâminas de $0,25 \mathrm{~m}$ a $0,46 \mathrm{~m}$ e apresentaram ajuste perfeito da reta entre os dados observados e os calculados (1:1), com $\mathrm{R}^{2}$ de 1,0 .

\section{Calibração dos equipamentos de campo}

Nessa fase foram calibrados: i) a vazão de entrada gerada pelo reservatório; ii) a vazão de entrada gerada pelo caminhão pipa; iii) o vertedor de $120^{\circ}$ que registra as vazões de entrada no plano de infiltração; iv) as medidas dos sensores de nível instalados no plano de infiltração.

Foram realizados três ensaios de campo para verificar a qualidade das medidas de nível de água realizada por sensores instalados a montante do vertedor de $120^{\circ}$ e no interior do plano de infiltração. Avaliaram-se também as medidas de vazão de entrada no plano obtidas pelo vertedor de $120^{\circ}$. Adicionalmente, registrou-se por meio fotográfico a área de inundação no interior do plano de infiltração. Para referência de área inundada, o plano foi estaqueado em linhas paralelas à canaleta de brita distribuidora e distanciadas de $3 \mathrm{~m}$. Marcaram-se as estacas a 7 $\mathrm{cm}$ acima do solo para conseguir visualizar as lâminas acima e abaixo deste valor no interior do plano.

As vazões dos ensaios foram produzidas pelo esvaziamento controlado de reservatórios, conforme figura 3. A descrição da medição dessas vazões é apresentada a seguir.

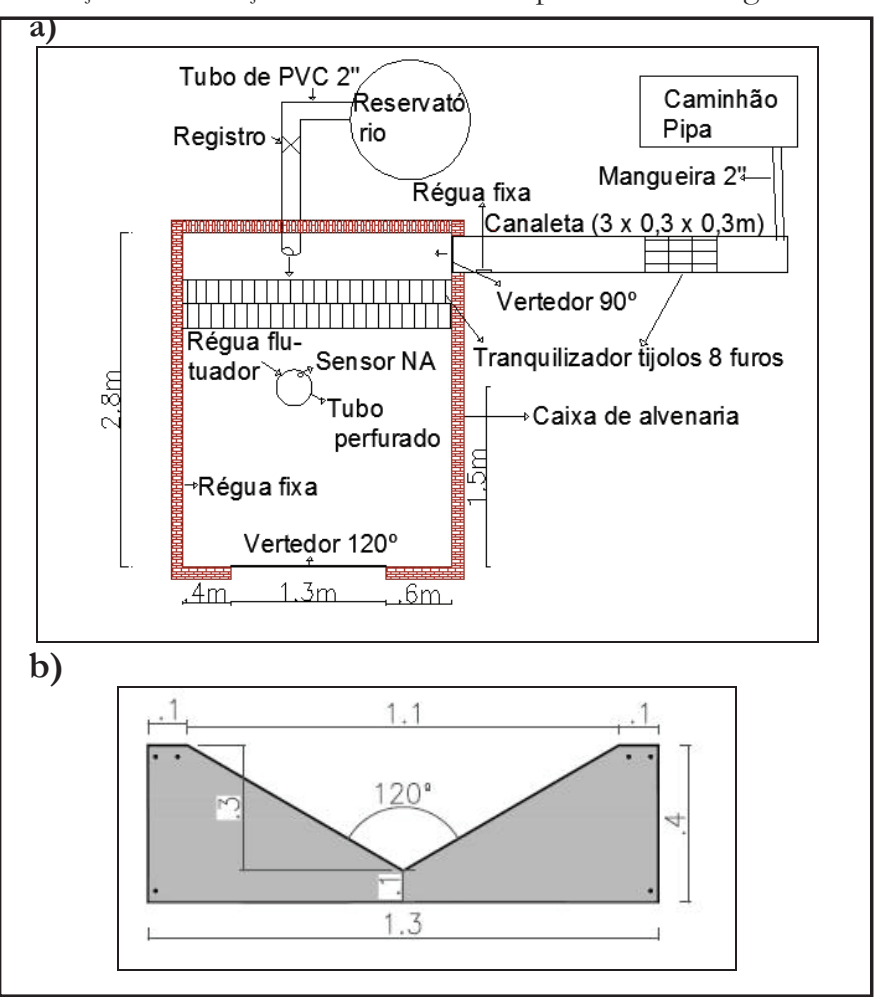

Figura 3- a) Esquema de ensaio de campo com chuva simulada. b) vertedor triangular de $120^{\circ}$ (medidas em metros)

\section{Vazão gerada pelo reservatório de $5000 \mathrm{~L}$}

A vazão gerada pelo reservatório de $5000 \mathrm{~L}$ foi obtida através do seu esvaziamento, conforme esquema da figura $3 \mathrm{a}$. A água, ao sair da caixa de água, foi conduzida para o plano por meio de um tubo de PVC de 2". Os níveis de água e o tempo foram medidos a intervalos de 1 minuto, com sensor de nível OTT, modelo Orpheus Mini. O hidrograma de esvaziamento foi obtido anteriormente por Felipe (2014), sob as mesmas condições usadas nesse experimento.

\section{Vazão gerada pelo caminhão pipa}

A vazão gerada pelo caminhão pipa durante o esvaziamento foi conduzida até a canaleta por meio de uma mangueira flexível de 2", conforme esquema da figura 3a. Nesta canaleta, após o tranquilizador, instalou-se uma régua graduada fixada na parede para medição dos níveis de água com leitura manual. No final da canaleta montou-se um vertedor triangular de $90^{\circ}$ de parede fina. Este vertedor foi calibrado em laboratório por Barbassa e Campos (2010), e se verificou que a equação proposta por Thomson (Equação 4) apresentou $\mathrm{R}^{2}$ de 0,99 para altura da crista em relação ao fundo de $5 \mathrm{~cm}$. Assim, realizaram-se leituras de nível de água a intervalos de 1 minuto para elaboração do hidrograma.

$$
Q=1,4 b^{2,5}
$$

Sendo $\mathrm{Q}$ a vazão $\mathrm{em}^{3}$ / $\mathrm{s}$ e h a lâmina sobre a crista do vertedor (m).

\section{Verificação em campo das medidas de vazões pelo vertedor de $120^{\circ}$ na entrada do plano de infil- tração}

As vazões de ensaio correspondem à soma do hidrograma produzido pelo esvaziamento do reservatório de água de 5000 L com o hidrograma gerado pelo esvaziamento do caminhão pipa. Essas vazões já aferidas foram usadas para verificação das medidas do vertedor triangular de $120^{\circ}$.

\section{Verificação das medidas de lâminas de água na crista do vertedor de $120^{\circ}$ pelo sensor de nível}

As lâminas de água na crista do vertedor na entrada do plano de infiltração foram registradas através de um sensor de nível OTT modelo Orpheus Mini, as quais foram aferidas duas réguas linimétricas, uma instalada no interior do tubo de PVC e a outra fixada na parede da caixa de alvenaria. Ambas as réguas foram lidas manualmente por dois operadores. Os pontos de instalação do sensor eletrônico e réguas são mostrados na figura 4(a).

Instalou-se também uma régua no interior do tubo de medida de NA no plano de infiltração para leitura manual e comparação com o registro automático do sensor OTT, conforme figura $2 \mathrm{a}$. 


\section{Modelagem do Plano de Infiltração}

Para a realização da modelagem foi utilizado o método de PULS. Esse método é um dos mais conhecidos na simulação da propagação de cheia em reservatório. O PULS utiliza a equação da continuidade (Equação 5) em que, a partir do hidrograma de entrada e valores iniciais de vazão e volume e das relações nível de água-vazão-volume, são obtidas as vazões de saída e o volume de armazenamento na estrutura para cada intervalo de tempo (CUDWORTH JÚNIOR, 1989).

Este método já foi aplicado por Barbassa, Angelini Sobrinha e Moruzzi (2014), Lucas, Barbassa e Moruzzi (2013) e Vieira, Silva e Nascimento (2009), entre outros.

A canaleta de distribuição e o plano de infiltração foram modelados como estruturas em série pelo método de PULS.

$$
Q e-Q s=d V / d t
$$

Onde: Qe é a vazão de entrada $\left(\mathrm{m}^{3} / \mathrm{s}\right)$; Qs é a vazão de infiltração $\left(\mathrm{m}^{3} / \mathrm{s}\right) ; \mathrm{V}$ é o volume de água reservado $\left(\mathrm{m}^{3}\right)$; e to tempo (s).

A variação do volume armazenado obtido pela integração da equação 5 é apresentado na equação 6 (BAPTISTA; NASCIMENTO; BARRAUD, 2005).

$$
\int_{V_{t}}^{V_{t+1}} d V=\int_{t}^{t+1} Q e(t) \cdot d t-\int_{t}^{t+1} Q s(t) \cdot d t
$$

A Equação 6 pode ser reescrita em diferenças finitas e reorganizada de forma a isolarem-se as incógnitas $\mathrm{V}_{\mathrm{t}+1}$ e $\mathrm{Q}_{\mathrm{t}+1}$ no primeiro membro da equação 7 (BAPTISTA; NASCIMENTO; BARRAUD, 2005; CHOW; MAIDMENT; MAYS, 1988).

$$
\frac{2 V_{t+1}}{\Delta t}+Q s_{t+1}=Q e_{t}+Q e_{t+1}-Q s_{t}+\frac{2 V_{t}}{\Delta t}
$$

Para o plano de infiltração desse trabalho, foram determinadas as relações entre níveis de água e vazões de saída e volumes reservados para a canaleta de distribuição, conforme segue.

Enquanto o valor de NA $\leq 0,30 \mathrm{~m}$ e enquanto houver vazão de entrada, haverá vazão de saída apenas para o solo da canaleta de distribuição, como aponta a equação 8 .

$$
Q s_{c}=K_{\text {sat }} \cdot A_{\text {inf } c}(N A) \text { para NA } \leq 0,3 \mathrm{~m}
$$

Onde: Qs é a vazão infiltrada pelas paredes e fundo da canaleta $\left(\mathrm{m}^{3} / \mathrm{s}\right) ; K_{\text {sat }}$ é a capacidade de infiltração saturada do solo (m/s); $\mathrm{A}_{\text {infc }}$ (NA) a área de infiltração da canaleta de distribuição, sendo essa representada pela Equação 9.

$$
A_{\text {info }}(\mathrm{N} A)=(2 \cdot \mathrm{NA}+0,4) .25
$$

Para NA $>0,3 \mathrm{~m}$ inicia-se o escoamento para o plano de infiltração propriamente dito, cuja vazão pode ser determinada considerando um vertedor retangular com largura igual a extensão da vala de distribuição de 25 m, conforme Porto (2005). A vazão de saída total da canaleta (Qst) é igual a Qsc somada ao escoamento para o plano (Qsp), conforme Equação 10.

$$
Q s t=Q s_{c}+Q s_{p}=K_{s a t} \cdot A_{\mathrm{inf}}(N A)+C \cdot L \cdot h^{1,5}
$$

Onde: $Q_{p}$ é a vazão extravasada para o plano de infiltração $\mathrm{em} \mathrm{m}^{3} / \mathrm{s}$, h é a lâmina acima da crista, que está a $0,3 \mathrm{~m}$ do fundo da trincheira (NA-0,3) m e C é o coeficiente básico de vazão, no caso o valor 2 foi adotado (PORTO, 2005).

O volume armazenado $(\mathrm{Vc})$ na canaleta de distribuição foi calculado pela Equação 11 .

$$
V c=B_{f} \cdot L_{p} \cdot N A
$$

Onde: $B_{f}$ é a largura do fundo igual a $0,4 \mathrm{~m}, L_{p} \mathrm{O}$ comprimento da canaleta igual a $25 \mathrm{~m}$ e $N A$ a lâmina de água dentro da canaleta em $\mathrm{m}$.

Com o emprego das Equações 8, 10 e 11 construíram-se as relações entre a cota do NA e as vazões de saída e os volumes armazenados e, portanto, de $2 . V / \Delta t+Q s$ para a canaleta de distribuição. Então, conhecido 2. $V / \Delta t+Q s$ pela Equação 7, pode-se calcular os valores correspondentes de $V$ e de Qst e, portanto, o valor de Qsc e de $Q s p$. A solução conjunta e iterativa destas relações e da Equação 7 possibilitou obter o hidrograma de entrada no plano de infiltração $Q e$.

Para simplificar a construção das relações entre cotas, vazões e volumes armazenados no plano de infiltração, considerou-se a condutividade do solo variável, porém em dois patamares, sendo eles: um até a condutividade hidráulica alcançar a fase assintótica, representada pela média neste intervalo de tempo; outro a Ksat. Então, até a condutividade hidráulica atingir a fase assintótica, foi empregada Equação 12.

$$
Q s_{p}=K_{i n i} \cdot A_{\text {inf }}(N A)
$$

Onde: $Q_{s \phi}$ é a vazão de saída do plano de infiltração $\left(\mathrm{m}^{3} / \mathrm{s}\right) ; K_{i n i}$ é a condutividade hidráulica média calculada do tempo 0 até o momento em que a condutividade torna-se constante; $A_{\text {inf }}$ a área de infiltração do plano $\left(\mathrm{m}^{2}\right)$ função da cota do $N A$ no ponto mais baixo do plano. Após este momento foi empregada a Equação 13.

$$
Q s_{p}=K_{\text {sat }} \cdot A_{\text {inf }}(N A)
$$

Onde: $K_{\text {sat }}$ é a condutividade hidráulica saturada $(\mathrm{m} / \mathrm{s})$.

O volume de água armazenado no plano (V) foi calculado conforme Equação 14.

$$
V=A_{\text {inf }}(N A) \cdot N A
$$

Com as equações 12, 13 e 14 e as restrições de tempo impostas construíram-se as relações entre cota do NA, vazões de 
saída e volumes e, portanto, o valor correspondente a $2 . \mathrm{V} / \Delta$ $t+Q s$ para o plano de infiltração.

Para medida de ajuste entre os níveis de água no plano de infiltração observados e modelados foram utilizados o coeficiente de determinação $\left(\mathrm{R}^{2}\right)$ e o coeficiente de Nash-Sutclife (NS), conforme Viessman Junior, Lewis e Knapp (1989).

A aplicação do modelo envolveu a calibração e a validação dos resultados medidos e calculados. Para iniciar a calibração adotaram-se os valores de condutividades $\left(k_{i n i}\right.$ e $\left.k_{\text {sat }}\right)$ determinadas em ensaio de Duplo Anel. Para valiação foram empregados valores médios de $K_{i m i}$ e $K_{s a t}$ obtidos na calibração.

Calcularam-se ainda os tempos de retardamento (Tret), de pico (Tpico), de escoamento no plano de infiltração (Tplano) e o coeficiente de escoamento superficial (C) para os eventos reais. O Tret foi computado como sendo a diferença entre os centros de massa do hietograma e do linigrama, pois não se dispõe de hidrograma de saída observado. O Tpico foi obtido pela diferença entre início da chuva e o pico de nível de água no plano. O Tplano foi contabilizado a partir do instante em que se iniciaram as vazões para o plano até o momento em que se iniciaram os registros de nível de água no ponto mais baixo do plano. O tempo de esvaziamento (Tesv) observado corresponde à diferença entre o instante em que ocorre o máximo nível e o instante de esvaziamento da estrutura. $\mathrm{O}$ mesmo pode ser determinado a partir do linigrama calculado por meio do método de PULS.

As Equações 15, 16 e 17 descrevem as relações utilizadas para determinação dos tempos descritos anteriormente.

$$
\text { Tret } \left.=\Sigma\left(T_{N A j} \cdot N A_{j}\right) / \Sigma N A j-\Sigma\left(T_{h i} \times h_{i}\right) / \Sigma h_{i}\right)
$$

Sendo: $T_{h i}$ o tempo i (min) em que ocorre a altura de chuva hi; $T_{N A j}$ o tempo $\mathrm{j}$ (min) em que ocorre a cota do nível de água $N A$ registrado no ponto mais baixo do plano.

$$
\text { Tpico }=T_{b 1}-T_{\text {NAmax }}
$$

Sendo: $T_{b 1}$ o tempo (min) em que ocorre a altura inicial de chuva no intervalo $1 . T_{N A \max }$ é o tempo (min) em que ocorre o máximo nível registrado.

$$
\text { Tplano }=\left(T_{Q 1}-T_{N A 1}\right)
$$

Sendo: $T_{01}$ o tempo (min) em que a vazão começa a escoar no plano; $\widetilde{T}_{N A 1}$ o tempo (min) em que ocorre o início de registro no ponto mais baixo do plano. O coeficiente de escoamento superficial foi determinado pelo quociente entre os volumes escoados (Vesd) e precipitados (Vch), ambos medidos, conforme Equação 18.

$$
C=V e s d / V c h
$$

O monitoramento do escoamento superficial e da formação de lâmina foi feito por meio de registros fotográficos de 113 fotos do plano, desde o início do ensaio até o desaparecimento da lâmina formada na superfície do plano.

\section{RESULTADOS E DISCUSSÕES}

\section{Verificação das medidas de lâminas em campo a montante do vertedor de $120^{\circ}$}

Foram obtidos três linigramas, sendo dois por meio de registros manuais nas réguas e um por meio do sensor OTT, para cada um dos três ensaios. Apresentam-se os linigramas do ensaio 2 na figura 4 .

Na tabela 1 são apresentados os erros absolutos médios e erros máximos entre as lâminas medidas automaticamente e as duas lâminas, registradas uma com régua fixa na parede e outra no interior do tubo de PVC. Verificou-se que os erros médios absolutos foram menores que $4 \mathrm{~mm}$.

Observou-se que os zeros do sensor de nível e das réguas utilizadas, que correspondem ao nível de água da cota da crista do vertedor de $120^{\circ}$, mostraram valores menores que $1 \mathrm{~mm}$.

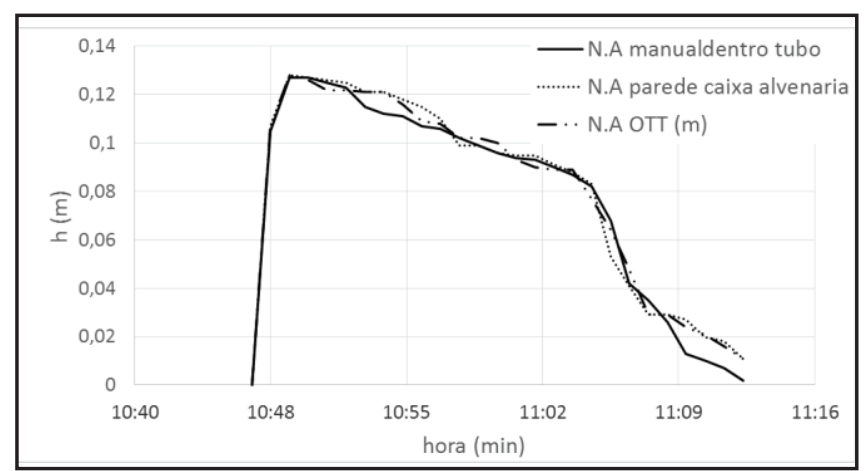

Figura 4 - Lâminas registradas manualmente por réguas (linha azul e laranja) e registradas pelo sensor de nível OTT (linha cinza) referente ao ensaio 2

Tabela 1 - Erros entre medidas dos níveis de água pelo sensor OTT e pelas réguas fixadas na parede e no tubo de PVC perfurado localizado a montante do vertedor de $120^{\circ}$

\begin{tabular}{ccccc}
\hline Ensaio & $\begin{array}{c}\text { Erro médio } \\
\text { absoluto } \\
\text { régua fixa } \\
(\mathrm{mm})\end{array}$ & $\begin{array}{c}\text { Erro } \\
\text { máx. } \\
\text { régua } \\
\text { fixa } \\
(\mathrm{mm})\end{array}$ & $\begin{array}{c}\text { Erro médio } \\
\text { absoluto } \\
\text { régua PVC } \\
(\mathrm{mm})\end{array}$ & $\begin{array}{c}\text { Erro } \\
\text { máx. } \\
\text { régua } \\
\text { PVC } \\
(\mathrm{mm})\end{array}$ \\
\hline 1 & 3 & 19 & 3 & 7 \\
2 & 2 & 11 & 2 & 11 \\
3 & 4 & 13 & 4 & 10 \\
\hline
\end{tabular}

\section{Verificação das vazões medidas pelo vertedor de $120^{\circ}$}

Foram obtidos dois hidrogramas, um pelo vertedor triangular de $120^{\circ}$ e o outro para as vazões descarregadas do caminhão pipa e do reservatório, para cada um dos três ensaios. Apresentam-se, a título de exemplo, os hidrogramas gerados no ensaio 2 na figura 5 .

$\mathrm{Na}$ tabela 2 apresentam-se os erros absolutos médios e erros máximos entre as vazões medidas pelo vertedor de $120^{\circ}$ (Qv) e as vazões resultantes dos esvaziamentos simultâneos 
do reservatório e do caminhão pipa $\left(Q_{e s v}\right)$. A diferença entre os volumes produzidos pelos esvaziamentos do reservatório e caminhão $\left.\left(\sum(\mathrm{Q} e s v) . \Delta \mathrm{t}\right)\right)$ em relação ao volume obtido pelo vertedor $\left.\left(\sum(Q v) . \Delta \mathrm{t}\right)\right)$ variou de 2,1 a $12 \%$ ou erro médio relativo de $7,1 \%$.

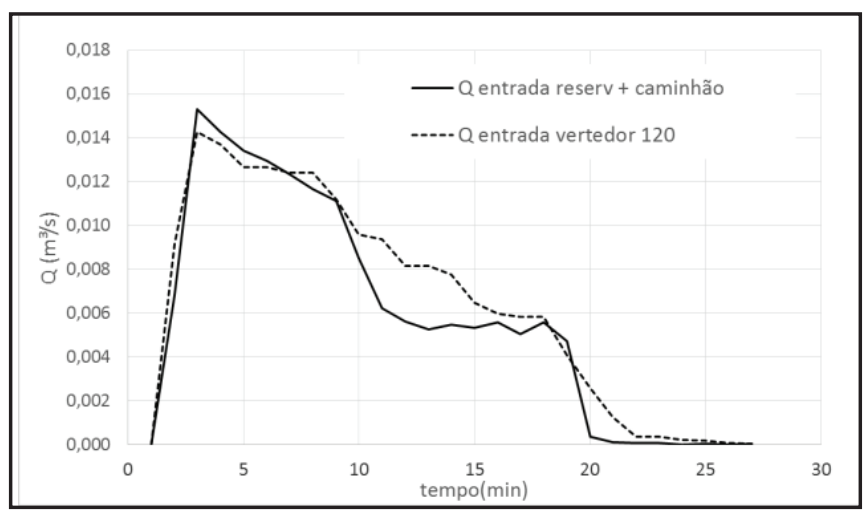

Figura 5 - Hidrograma gerado pelo esvaziamento da caixa de água e caminhão pipa (linha azul) e medido pelo vertedor de $120^{\circ}$ (linha amarela) referente ao ensaio 2

Tabela 2 - Erros determinados entre vazões e volumes medidos no vertedor de $120^{\circ}\left(Q_{V}\right.$ e $\left.V\right)$ e pelo esvaziamento do reservatório e do caminhão pipa ( $V_{V}$ e $\left.V_{e s s}\right)$

\begin{tabular}{|c|c|c|c|c|}
\hline .ำ. & $\begin{array}{c}\left\|Q_{v}-Q_{e s v}\right\| \\
\text { médio } \\
\left(\mathrm{m}^{3} / \mathrm{s}\right)\end{array}$ & $\begin{array}{c}\left\|Q_{v}-Q_{e s v}\right\| \\
\text { máximo } \\
\left(\mathrm{m}^{3} / \mathrm{s}\right)\end{array}$ & $\begin{array}{c}\left(V_{v}-V_{\text {esv }}\right) \\
\left(\mathrm{m}^{3}\right)\end{array}$ & $\begin{array}{c}\left\|V_{V}-V_{e s v}\right\| \\
\text { por } \\
\mathbf{V}_{\text {esv }} \\
(\%)\end{array}$ \\
\hline 1 & 0,061 & 0,242 & 0,198 & 2,1 \\
\hline 2 & 0,084 & 0,549 & 1,124 & 12,0 \\
\hline 3 & 0,065 & 0,293 & 0,656 & 7,2 \\
\hline
\end{tabular}

\section{Comportamento hidrológico do plano de infiltra- ção durante os ensaios de campo}

Por meio do registro fotográfico do ensaio 2 , mostrado na figura 6 , constatou-se o seguinte: o escoamento teve início às 10:52, com máxima área com lâmina às 10:59 e às 11:16 não se visualizou lâmina sobre a superfície do plano, o que permite estimar tempo de funcionamento (Df) de $24 \mathrm{~min}$. Os dados dos demais ensaios são apresentados na tabela 3. Estimou-se a área de inundação máxima média (presença visual de lâmina) nos três ensaios de aproximadamente $90 \mathrm{~m}^{2}(9+27+27+18+9)$.

O tempo adotado para a condutividade atingir a fase assintótica foi igual a $14 \mathrm{~min}$, tal como obtido em ensaios de duplo anel em triplicada, conforme mostrado na figura 5 .

Observa-se na figura 6 que $K_{\text {sat }}$ converge ao valor de 180 $\mathrm{mm} / \mathrm{h}$ após cerca de 14 minutos. A condutividade inicial média $\left(K_{i n i}\right)$ entre 0 e $14 \mathrm{~min}$ foi igual a $356 \mathrm{~mm} / \mathrm{h}$. A representação da condutividade em dois patamares é mostrada na mesma figura 2 , tendo sido esta empregada na modelagem.

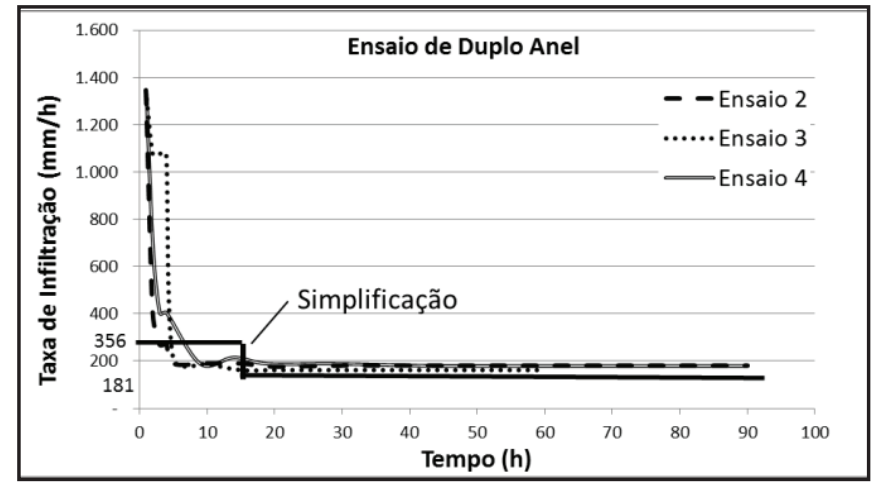

Figura 6 - Taxas de infiltração obtidas por meio dos ensaios de Duplo Anel, em triplicada, e com representação simplificada em dois patamares

A modelagem da valeta de distribuição pelo método de PULS com Ksat obtido pelo ensaio de duplo anel $(180 \mathrm{~mm} / \mathrm{h})$ possibilitou estimar que, em média, $3,1 \mathrm{~m}^{3}$ são infiltrados via canal de distribuição e $6,2 \mathrm{~m}^{3}$ extravazam para o plano de infiltração. Observaram-se destes ensaios o que segue:

- O escoamento pela superfície do plano ocorre em áreas preferenciais, provavelmente devido aos desníveis na superfície gramada do plano, conforme figura 7.

- Estima-se que as lâminas escoadas sejam menores que $6 \mathrm{~cm}$, pois não atingiram marcas feitas nas estacas de referência.

- Comprova-se que escoamento de $6,2 \mathrm{~m}^{3}$ não alcança o final do plano. Isto se deve à retenção e detenção superficial, bem como às altas taxas de infiltração verificadas em ensaios de duplo anel, após recuperação da superfície por escarificação.

Presume-se então que a relação cota-volume-vazão necessária para aplicação do método de PULS não é representativa para vazões e volumes de entrada desta ordem, pois não há registro de lâmina. Isto pode ser estendido ao início do escoamento de chuvas mais intensas. Outra dificuldade que ocorre ao tentar representar o comportamento dos níveis por meio do método de PULS aplicado ao plano de infiltração é a variação da área inundada devido a pequenos desníveis da superfície do plano que causam grandes variações de área.

\section{Monitoramento e Funcionamento do Plano de Infiltração}

Os ensaios de granulometria indicaram a classificação do solo local como areia média a fina argilosa. O monitoramento das precipitações, vazões afluentes e dos níveis de água no plano de infiltração foi realizado de 16/12/2013 a 18/03/2014. Neste período foram registrados 32 eventos de chuva, dos quais 7 apresentaram nível de água registrado pelo sensor de nível no interior do plano e cujos dados são mostrados na tabela 4.

Verifica-se que o evento 2 com o maior valor de $\mathrm{Tr}$ registrado entre os ensaios (6,1 anos) e maior lâmina máxima $(0,24 \mathrm{~m})$ não ultrapassou a profundidade máxima de $0,59 \mathrm{~m}$. O evento 7 assemelha-se aos ensaios de campo com reservatório e caminhão, pois seu volume escoado foi de $10,4 \mathrm{~m}^{3}$, porém precedido 2 h pelo evento 6 com $43,2 \mathrm{~mm}$, o que pode justificar a presença de lâmina no plano, registrada pelo sensor. Para os 
eventos 1 , 4 e 5 verificaram-se precipitações antecedentes de 22,8 $\mathrm{mm}, 28,6 \mathrm{~mm}$ e 4,3 $\mathrm{mm}$ respectivamente.

Apresentam-se na tabela 5 os tempos de retardamento, de pico e também o tempo decorrido entre o momento de início de escoamento para o plano e o momento de registro do nível pelo sensor no final do plano (Tplano).

O Tplano talvez seja o mais representativo, porque é o tempo do escoamento sobre o plano propriamente dito (com resultado médio de 12,2 min). Neste, não são contabilizados os tempos de escoamento no telhado e na rede predial, presentes no Tpico (com resultado médio de 28,3 min). Essas medidas de tempo evidenciam importantes efeitos da detenção superficial, exatamente no período em que a condutividade hidráulica assume seu maior valor, capaz até de evitar acúmulo de água no final do plano.

Verifica-se que o coeficiente de escoamento do telhado metálico, em média, foi de 0,83 e se excluído o menor dos eventos

Tabela 3 - Volumes lançados e calculados pelo vertedor de $120^{\circ}$, área inundada e tempo de funcionamento resultantes dos ensaios de campo (Df)

\begin{tabular}{|c|c|c|c|c|}
\hline Ensaio & $\begin{array}{c}\text { Volume } \\
\text { lançado } \\
\left(\mathrm{m}^{3}\right)\end{array}$ & $\begin{array}{l}\text { Vol. calc. } \\
\text { Vert. } 120^{\circ} \\
\left(\mathrm{m}^{3}\right)\end{array}$ & $\begin{array}{l}\text { Área } \\
\text { inun } \\
\left(\mathrm{m}^{2}\right)\end{array}$ & $\underset{(\mathrm{min})}{D f}$ \\
\hline 1 & 9,6 & 9,8 & -90 & $\begin{array}{c}25 \\
(09: 31 \mathrm{a} \\
09: 56)\end{array}$ \\
\hline 2 & 9,3 & 10,4 & -90 & $\begin{array}{c}24 \\
(10: 52 a \\
11: 16)\end{array}$ \\
\hline 3 & 9,1 & 9,7 & -90 & $\begin{array}{c}28 \\
(12: 08 \mathrm{a} \\
12: 36)\end{array}$ \\
\hline
\end{tabular}

Tabela 4 - Alturas, durações, intensidade, períodos de retorno, volumes precipitados e escoados e altura máxima de água dos eventos reais monitorados

\begin{tabular}{|c|c|c|c|c|c|c|c|}
\hline$\dot{\mathrm{Z}}$ & $\begin{array}{l}\widehat{E} \\
\text { ह્ } \\
2\end{array}$ & હ્રી & 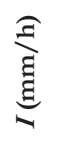 & 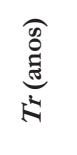 & 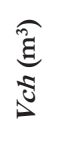 & 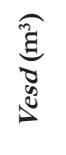 & 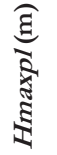 \\
\hline 1 & 45,0 & 4,7 & 9,5 & 2,4 & 78,6 & 73,7 & 0,18 \\
\hline 2 & 43,2 & 1,1 & 39,2 & 6,1 & 75,6 & 52,9 & 0,24 \\
\hline 3 & 28,6 & 0,4 & 3,3 & 4,1 & 50,1 & 38,7 & 0,17 \\
\hline 4 & 21,4 & 6,5 & 68,7 & 0,03 & 37,4 & 33,1 & 0,14 \\
\hline 5 & 18,7 & 1,1 & 16,3 & 0,07 & 31,8 & 25,7 & 0,12 \\
\hline 6 & 17,1 & 0,4 & 68,8 & 0,32 & 29,8 & 29,0 & 0,12 \\
\hline 7 & 7,9 & 1,0 & 8,3 & 0,005 & 13,8 & 10,4 & 0,05 \\
\hline
\end{tabular}

Tabela 5 - Tempos de retardo, pico, de escoamento no plano e coeficiente de escoamento do telhado

\begin{tabular}{ccccc}
\hline $\begin{array}{c}\text { No. } \\
\text { evento }\end{array}$ & $\begin{array}{c}\text { Tret } \\
(\mathrm{min})\end{array}$ & $\begin{array}{c}\text { Tpico } \\
(\mathrm{min})\end{array}$ & $\begin{array}{c}\text { Tplano } \\
(\mathrm{min})\end{array}$ & $\begin{array}{c}\boldsymbol{C} \text { te- } \\
\text { lhado }\end{array}$ \\
\hline 1 & 2,7 & 26 & 4,8 & 0,94 \\
2 & 6,3 & 36 & 11,9 & 0,70 \\
3 & 9,9 & 38 & 9,5 & 0,77 \\
4 & 16,4 & 32 & 13,7 & 0,88 \\
5 & 0,3 & 19 & 18,2 & 0,97 \\
6 & 1,4 & 20 & 16,3 & 0,82 \\
7 & 0,9 & 27 & 11,1 & 0,76 \\
Médias & $\mathbf{5 , 9}$ & $\mathbf{2 8 , 3}$ & $\mathbf{1 2 , 2}$ & $\mathbf{0 , 8 3}$ \\
\hline
\end{tabular}

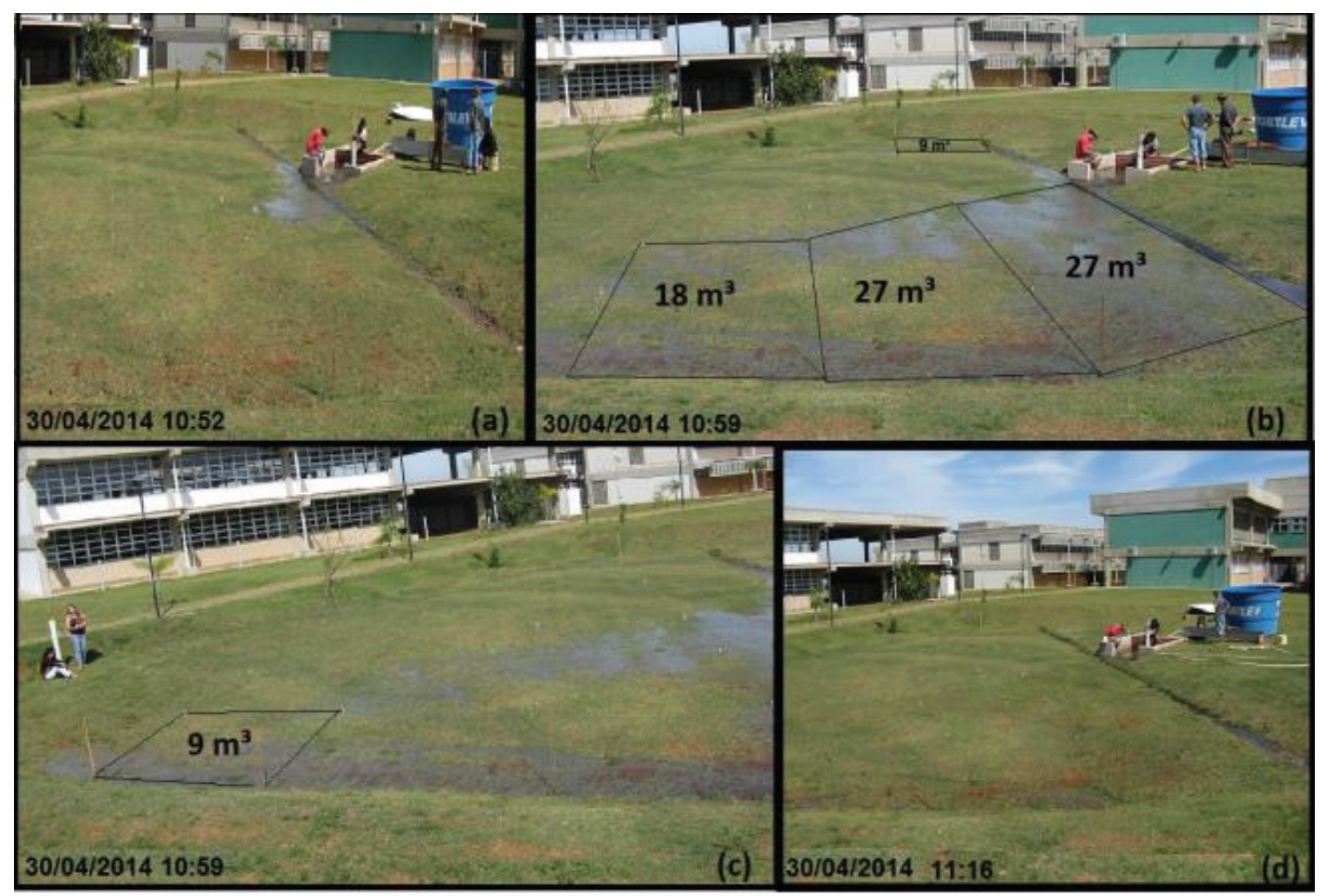

Figura 7 - Ensaio de campo 2 com caixa de água e caminhão pipa: (a) início do ESD no plano (10:52), (b) e (c) momento com máxima área molhada (10:59), (d) final da infiltração no plano (11:16) 
0,85 , portanto valores dentro da faixa da literatura.

\section{Modelagem pelo método de PULS}

Os eventos 1, 3, 5 e 7 foram utilizados para calibração do modelo de PULS, variando em $20 \%$ as condutividades, para mais ou menos, em relação aos resultados médios obtidos dos ensaios de duplo anel (Kini de $356 \mathrm{~mm} / \mathrm{h}$ e Ksat de $181 \mathrm{~mm} / \mathrm{h}$ ).

Mostram-se na tabela 6 os ajustes do PULS com condutividade variável simplificada e com Ksat, obtidos por calibrações manuais. As modelagens com condutividade variável apresentaram leve melhora na aderência aos dados observados relativamente às modelagens com Ksat, conforme se observa na mesma tabela 6 .

Podem-se considerar estes resultados razoáveis, na medida em que a área inundada e a infiltração ocorrida até o momento que a água chega ao ponto mais baixo do plano não são registradas, ou seja, não são computadas pelo PULS, como se demonstrou com o ensaio de campo com reservatório e caminhão pipa. O não cômputo da área inundada inicial e as altíssimas infiltrações iniciais observadas em ensaio de duplo anel (média de 354,1 mm/h) fazem com que as vazões iniciais calculadas pelo PULS sejam bem maiores que aquelas observadas, como se demonstra na figura 8 .

Tabela 6 - Calibrações do modelo de PULS aos eventos reais com condutividade variável simplificada e com condutividade constante Ksat

\begin{tabular}{cccccc}
\hline \multirow{2}{*}{ No. } & \multicolumn{2}{c}{$\begin{array}{c}\text { Condutividade } \\
\text { variável simpl. }\end{array}$} & $\begin{array}{c}\text { Condutividade } \\
\text { constante }\end{array}$ \\
\cline { 2 - 6 } & $\begin{array}{c}\text { Kini } \\
\text { Ksat } \\
(\mathrm{mm} / \mathrm{h})\end{array}$ & $\begin{array}{c}\mathrm{R}^{2} \\
\mathrm{NS}\end{array}$ & $\begin{array}{c}\text { Ksat } \\
(\mathrm{mm} / \mathrm{h})\end{array}$ & $\begin{array}{c}\mathrm{R}^{2} \\
\mathrm{NS}\end{array}$ & $\begin{array}{c}\Delta \mathrm{R}^{2} \\
\Delta \mathrm{NS}\end{array}$ \\
\hline \multirow{2}{*}{1} & 326,4 & 0,86 & \multirow{2}{*}{153,0} & 0,84 & 0,02 \\
& 153,0 & 0,54 & & 0,52 & 0,02 \\
\hline \multirow{2}{*}{3} & 391,6 & 0,85 & \multirow{2}{*}{198,0} & 0,75 & 0,10 \\
& 198,0 & 0,92 & & 0,86 & 0,06 \\
\hline \multirow{2}{*}{5} & 427,2 & 0,56 & \multirow{2}{*}{216,0} & 0,55 & 0,01 \\
& 216,0 & 0,40 & & 0,39 & 0,01 \\
\hline \multirow{2}{*}{7} & 427,2 & 0,73 & \multirow{2}{*}{216,0} & 0,73 & 0,00 \\
& 216,0 & 0,48 & & 0,48 & 0,00 \\
\hline \multirow{2}{*}{ Média } & $\mathbf{3 9 3 , 1}$ & $\mathbf{0 , 7 5}$ & \multirow{2}{*}{$\mathbf{1 9 5 , 8}$} & $\mathbf{0 , 7 2}$ & $\mathbf{0 , 0 3}$ \\
& $\mathbf{1 9 5 , 8}$ & $\mathbf{0 , 5 9}$ & & $\mathbf{0 , 5 6}$ & $\mathbf{0 , 0 3}$ \\
\hline
\end{tabular}

Os ajustes obtidos pelas validações do modelo de PULS aos eventos 2, 4 e 6 são apresentados na tabela 7. A modelagem com Ksat em relação ao emprego de condutividade variável simplificada fez reduzir tanto $\mathrm{R}^{2}$ de até 0,16 e NS de até 0,39 , portanto, uma variação razoável. Exemplificam-se os resultados da validação pela apresentação do evento 6 , conforme figura 9 , a partir dos linigramas medidos e calculados.

O tempo de esvaziamento (Tesv) observado por meio do monitoramento da técnica foi, no máximo, de $150 \mathrm{~min}$ ou 2,5 h, muito aquém do recomendado de 24 h, conforme se observa na tabela 8 . Os tempos de esvaziamento calculados, considerando Kini e Ksat ou somente Ksat, foram praticamente iguais. A diferença entre os Tesv observado e calculado variaram entre 7 e $84 \%$, excluído o menor dos eventos monitorados (evento

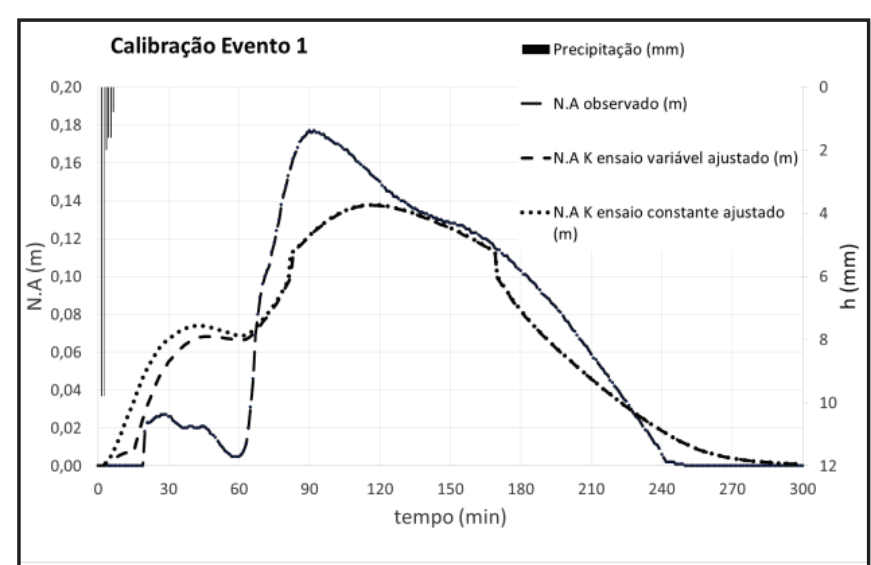

Figura 8 - Linigramas observados e calibrados por modelo de PULS com condutividade variável simplificada e constante (Ksat)

Tabela 7 - Ajustes das validações do modelo de PULS aos eventos reais 2,4 , e 6 com condutividade variável simplificada e com condutividade constante

\begin{tabular}{|c|c|c|c|c|c|}
\hline \multirow{3}{*}{ No. } & \multicolumn{2}{|c|}{$\begin{array}{l}\text { Condutividade } \\
\text { variável simpl. }\end{array}$} & \multicolumn{2}{|c|}{$\begin{array}{c}\text { Condutividade } \\
\text { constante }\end{array}$} & \multirow{3}{*}{$\begin{array}{l}\Delta \mathrm{R}^{2} \\
\Delta \mathrm{NS}\end{array}$} \\
\hline & Kini & $\mathrm{R}^{2}$ & \multirow{2}{*}{$\begin{array}{c}\text { Ksat } \\
(\mathrm{mm} / \mathrm{h})\end{array}$} & $\mathrm{R}^{2}$ & \\
\hline & $\begin{array}{l}\text { Ksat } \\
(\mathrm{mm} / \mathrm{h})\end{array}$ & NS & & NS & \\
\hline \multirow{2}{*}{2} & 393,1 & 0,65 & \multirow{2}{*}{195,8} & 0,59 & $-0,06$ \\
\hline & 195,8 & 0,50 & & 0,46 & $-0,04$ \\
\hline \multirow{2}{*}{4} & 393,1 & 0,92 & \multirow{2}{*}{195,8} & 0,92 & 0,00 \\
\hline & 195,8 & 0,95 & & 0,95 & 0,00 \\
\hline \multirow{2}{*}{6} & 393,1 & 0,78 & \multirow{2}{*}{195,8} & 0,62 & $-0,16$ \\
\hline & 195,8 & 0,88 & & 0,49 & $-0,39$ \\
\hline \multirow{2}{*}{\multicolumn{2}{|c|}{ Médias }} & 0,71 & & 0,71 & $-0,07$ \\
\hline & & 0,78 & & 0,63 & $-0,15$ \\
\hline
\end{tabular}

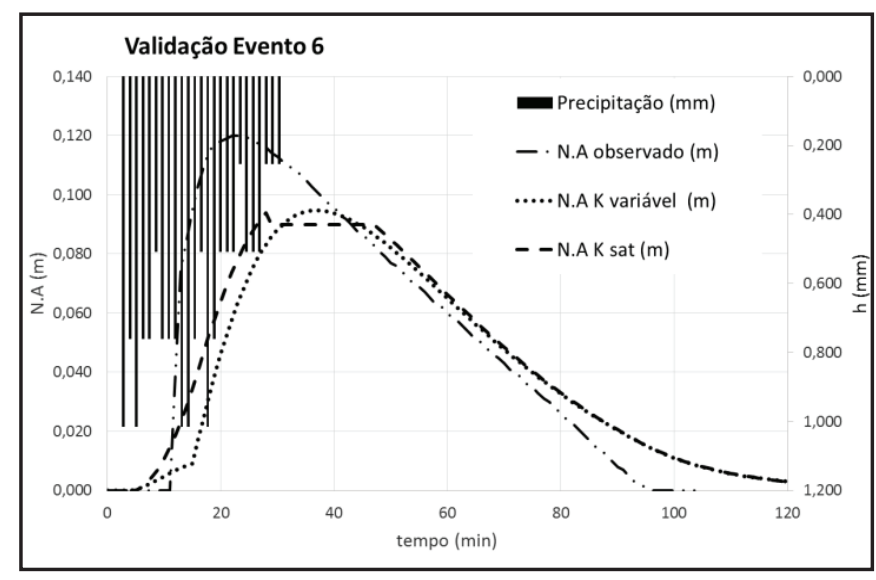

Figura 9 - Linigramas observado e calculados para validação da modelagem de PULS, com condutividade variável simplificada e constante (Ksat) 
7), conforme tabela 8 .

Tabela 8 - Tempo de esvaziamento (Tesv) calculado e observado

\begin{tabular}{cccc}
\hline Evento & $\begin{array}{c}\text { Tesv } \\
\text { observado } \\
\text { (min) }\end{array}$ & $\begin{array}{c}\text { Tesv } \\
\text { calculado } \\
\text { (min) }\end{array}$ & $\begin{array}{c}\text { Erro } \\
\mathbf{( \% )}\end{array}$ \\
\hline 1 & 150 & 161 & 7 \\
2 & 71 & 105 & 48 \\
3 & 112 & 142 & 26 \\
4 & 90 & 96 & 7 \\
5 & 46 & 85 & 84 \\
6 & 80 & 101 & 26 \\
7 & 20 & 60 & 200 \\
\hline
\end{tabular}

\section{CONCLUSÕES}

Os ensaios com esvaziamentos de reservatórios (5000 L e caminhão pipa) mostraram que a propagação pelo plano se faz em áreas preferenciais. Este fato, associado aos tempos de retardo, de pico e às taxas de infiltração, contribuiu para não haver registros de nível de água no plano de infiltração. Este efeito pode ser estendido para o início de precipitações, bem como para precipitações de pequenas lâminas.

As calibrações do modelo PULS com condutividade hidráulica variável e simplificada em dois patamares mostraram que a última apresenta resultados levemente melhores $\left(\mathrm{R}^{2}\right.$ médio de 0,75$)$ se comparada às calibrações com condutividade hidráulica saturada $\left(\mathrm{R}^{2}\right.$ médio de 0,72$)$. As validações do modelo apresentaram o mesmo comportamento, com $\mathrm{R}^{2}$ pouco menor, porém com valor do coeficiente de Nash-Sutcliffe maior.

O plano de infiltração, devido à sua geometria, em eventos de baixa altura pluviométrica tem seu volume distribuído irregularmente na área de infiltração, dificultando a representatividade da relação lâmina volume necessária ao método de PULS.

À grande área de infiltração desta técnica também são atribuídos baixos valores de tempo de esvaziamento, observados e calculados e, neste caso, aliada às altas taxas de infiltração.

\section{REFERÊNCIAS}

ABNT - ASSOCIAÇÃO BRASILEIRA DE NORMAS TÉCNICAS. NBR 6457: Preparação de amostras de solo para ensaios de compactação e ensaios de caracterização: método de ensaio. Rio de Janeiro: ABNT, 1986a.

ABNT - ASSOCIAÇÃO BRASILEIRA DE NORMAS TÉCNICAS. NBR 6502: Rochas e solos - Terminologia. Rio de Janeiro: ABNT, 1995.

ABNT - ASSOCIAÇÃO BRASILEIRA DE NORMAS TÉCNICAS. NBR 7181: Solo - Análise granulométrica. Rio de Janeiro: ABNT, 1984.

ABNT - ASSOCIAÇÃO BRASILEIRA DE NORMAS
TÉCNICAS. NBR 7182: Ensaio de Compactação. Rio de Janeiro: ABNT, 1986b.

ASCE. Design and Construction of Sanitary and Storm Sewers. New York: ASCE, 1969. (Manual and Reports of Engineering Practice, 37).

BAPTISTA, M.; NASCIMENTO, N.; BARRAUD, S. Técnicas compensatórias em drenagem urbana. 2. ed. Porto Alegre: ABRH, 2011.

BARBASSA, A. P.; ANGELINI SOBRINHA, S.; MORUZZI, R. B. Poço de infiltração para controle de enchentes na fonte: avaliação das condições de operação e manutenção. Ambiente Construído, Porto Alegre, v. 14, n. 2, p. 91-107, abr./jun. 2014.

BARBASSA, A. P.; CAMPOS, J. B. N. de. Comportamento Hidrológico de Áreas Urbanas Impermeabilizadas Diretamente Conectadas e Total. RBRH: revista brasileira de recursos hídricos, v. 15, n. 4, p. 69-79, out./dez. 2010.

BOURGOGNE, P. 25 ans de solutions compensatoires d'assainissement pluvial sur la communauté urbaine de Bordeaux. [S.1.]: NOVATECH, 2010.

CHAHAR, B. R.; GRAILLOT, D.; GAUR, S. Stormwater management through infiltration trenches. J Irrigation Drainage Eng., ASCE, v. 138, n. 3, p. 274-281, 2012.

CHOW, V. T.; MAIDMENT D. R.; MAYS, L. W. Applied Hydrology. New York: McGraw-Hill, 1988.

CUDWORTH JÚNIOR, A. G. Flood hydrology manual. A water Resources Technical Publication. Denver, Co: U.S. Departament of Interior, Bureau of Reclamation, 1989.

DECHESNE, M.; BARRAUD, S.; BARDIN, J. Experimental assessment of stormwater infiltration basin evolution. J. Environ. Eng., ASCE, v. 131, n. 7, p. 1090-1098, July 2005.

FUAMBA, M.; WALLISER, T.; DAYNOU, M.; ROUSSELLE, J. Methodology selection of best management practices and economic analysis: a case study in Quebec, Canada: NOVATECH, 2010.

LUCAS, A. H. Monitoramento e Modelagem de um sistema Filtro Vala - Trincheira de infiltração em escala real. São Carlos. 2011. Dissertação (Mestrado em Engenharia Urbana) - Universidade Federal de São Carlos, São Carlos, 2011.

LUCAS, A. H.; BARBASSA, A. P.; MORUZZI, R. B. Modelagem de um sistema filtro-vala-trincheira de infiltração pelo método de PULS adaptado para calibração de parâmetros. RBRH: revista brasileira de recursos hídricos, v. 18, n. 2, p. 225-236, abr. 2013.

MARTIN, C.; RUPERD, Y.; LEGRET, M. Urban stormwater drainage management: the development of a multicriteria decision aid approach for best management practices. Eur. J. Oper. Res., v. 181, n. 1, p. 338-349, Aug. 2006. 
MOURA, T. A. M. Estudo experimental de superficies permeáveis para o controle de escoamento superficial em ambientes urbanos. 2005. Dissertação (Tecnologia Ambiental e Recursos Hídricos) - Universidade de Brasília, Brasília, 2005.

PORTO, R. M. Hidráulica Básica. São Carlos: EESC-USP, 2005.

REIS, R. P. A.; OLIVEIRA, L. H.; SALES, M. M. Sistemas de drenagem na fonte por poços de infiltração de água pluvial. Ambiente Construido, Porto Alegre, v. 8, n. 2, p. 99-117, abr./ jun. 2008.

SCHUELER, T. R. Controlling urban runoff: a practical manual for planning and designing urban BMPs. Washington: Department of Environmental Programs. Washington Metropolitan Water Resources Planning Board, 1987.

SOUZA, V. C. B. Estudo experimental de trincheiras de infiltração no controle da geração do escoamento superficial. 2002. 127 p. Tese (Doutorado em Engenharia de Recursos Hídricos e Saneamento Ambiental) - Universidade Federal do Rio Grande do Sul, Porto Alegre, 2002.

TUCCI, C. E. M. Água no Meio Urbano. In: TUCCI, C. E. M. Água Doce. Porto Alegre: Instituto de Pesquisas Hidráulicas, Universidade Federal do Rio Grande do Sul, 1997. cap. 14.

TUCCI, C. E. M.; PORTO, R. L.; BARROS, M. T. Drenagem urbana. Rio Grande do Sul: Editora da Universidade, 1995.

URBONAS, B. R. Assessment of Stormwater Best Management Practice Effectiveness. In: HEANEY, J. P.; PITT, R.; FIELD, R. Innovative Urban Wet-Weather Flow Management Systems. Cincinnati, OH: National Risk Management Research Laboratory, 1993. p. 7-1-7-46.

VIEIRA, L.; SILVA, A.; NASCIMENTO, N. O. Avaliação da Eficiência Hidráulica de Duas Técnicas Alternativas de Drenagem Urbana - Estudo de Caso de Um Trecho da Bacia do Mergulhão na Pampulha. In: SIMPÓSIO BRASILEIRO DE RECURSOS HÍDRICOS, 13., 2009, Campo Grande. Anais... Porto Alegre: ABRH, 2009.

VIESSMAN JUNIOR, W.; LEWIS, G. L.; KNAPP, J. K. Introduction to bydrology. 3. ed. New York: Haper e Row Publisher, 1989. 